used RNA interference-which inhibits gene expression-to show that reduction in SPINK1 expression in these cells reduced their invasiveness.

The researchers conclude that SPINK1overexpressing prostate cancers are an aggressive subset of ETS-negative cancers (which comprise $\sim 10 \%$ of prostate cancer cases) that can hopefully be detected, in future, by a urine test. Further research is required to understand the molecular mechanisms that underlie the aggressiveness of SPINK1-overexpressing prostate cancers.

Original article Tomlins SA et al. (2008) The role of SPINK1 in ETS rearrangement-negative prostate cancers. Cancer Cell 13: $519-528$

\section{LPN unsuitable for elderly patients with poor renal function}

Laparoscopic partial nephrectomy (LPN) is usually favored over open surgery to remove small renal masses. LPN, however, requires prolonged warm ischemia time, which has been associated with raised complication rates. In a new study, Colombo and colleagues investigated the effect of warm ischemia time during LPN on renal function in patients with pre-existing renal dysfunction.

They assessed 485 patients who underwent LPN at the Cleveland Clinic, 48 of whom had abnormal baseline renal function (serum creatinine level $\geq 88 \mu \mathrm{mol} / \mathrm{l}$ ). Compared with the 437 patients with normal renal function, patients with pre-existing renal dysfunction were older (mean age 67.6 years vs 58.6 years), had larger tumors $(3.3 \mathrm{~cm}$ vs $2.7 \mathrm{~cm}$ ), and had more comorbidities. Overall, the perioperative, postoperative, and renal function outcomes and complication rates were similar in both groups. Patients with inferior renal function before surgery had poorer estimated 5-year overall survival than those with normal renal function $(78 \%$ vs $90 \%, P=0.01)$, but similar cancer-specific survival (100\% vs $98 \%, P=0.65)$. Among the 48 patients with poor renal function, those aged $>70$ years and whose warm ischemia time was $>30$ min experienced a significant deterioration in serum creatinine and glomerular filtration rate compared with the rest of this group.
The authors conclude that nephron-sparing techniques other than LPN should be considered for elderly patients (>70 years) with compromised renal function, who are at high risk of renal dysfunction after LPN.

Original article Colombo JR Jr et al. (2008) Laparoscopic partial nephrectomy in patients with compromised renal function. Urology 71: 1043-1048

\section{Reduced fluid intake could benefit patients with overactive bladder syndrome}

First-line therapy for patients with overactive bladder syndrome (OBS) includes lifestyle interventions such as fluid-intake manipulation. Consensus is lacking on what type and extent of fluid-intake manipulation is most beneficial, however, so Hashim and Abrams assessed the effect of increased or decreased fluid intake on symptoms and quality of life in patients with OBS.

The prospective, crossover trial included 24 patients with OBS who were randomly allocated to one of two groups. In one group, manipulations were ordered as follows: $25 \%$ reduction, 50\% reduction, 25\% increase, 50\% increase (all relative to baseline fluid intake). Each manipulation lasted 4 days, separated by 2-day periods of normal fluid intake; the other group underwent these manipulations in reverse order. Patients also completed a validated quality-of-life questionnaire after each 4-day manipulation. Patients did not participate in manipulations that would have required them to drink $>3$ I or $<1$ I per day.

Significant reductions in daytime frequency, urgency and nocturia occurred with the $25 \%$ reduction in fluid intake. Symptoms also improved with the $50 \%$ reduction. By contrast, increasing fluid intake by $25 \%$ or $50 \%$ significantly worsened daytime frequency, but did not affect urgency or nocturia. Fluid manipulations did not affect overall quality of life, but the $25 \%$ reduction did produce subjective improvements in urgency and nocturia.

The findings suggest that patients with OBS could be advised to reduce their fluid intake by $25 \%$ to improve symptoms, as long as they do not drink <1 I per day.

Original article Hashim $\mathrm{H}$ and Abrams P (2008) How should patients with an overactive bladder manipulate their fluid intake? BJU Int 102: 62-66 